\title{
Zonal Colonic Aganglionosis, a Variant of Hirschsprung's Disease
}

\author{
A. G. MACIVER and R. WHITEHEAD \\ From the Department of Pathology, Radcliffe Infirmary, Oxford
}

\begin{abstract}
MacIver, A. G., and Whitehead, R. (1972). Archives of Disease in Childhood, 47, 233. Zonal colonic aganglionosis, a variant of Hirschsprung's disease. On a review of 15 cases of Hirschsprung's disease of the colon we have encountered 4 cases of an atypical variant. These 4 cases had a zone of aganglionosis of both intermyenteric and submucous plexuses in the sigmoid colon and, in 1 case, a further zone of aganglionosis in the rectum. Ganglion cells reappeared distal to the sigmoid zone of aganglionosis. A transition zone was not invariably present. The clinical features demonstrated a variability of presentation typical of the more usual form of the disease. The importance of atypical Hirschsprung's disease is discussed from a diagnostic and pathogenetic viewpoint.
\end{abstract}

Bodian (1960) with a personal experience of 251 cases of Hirschsprung's disease of the colon stated that the characteristic aganglionic segment always extended distally to the anal margin. Submucous rectal biopsy was advocated as the only suitable diagnostic procedure as it is based on direct observation of the fundamental pathology of this disease. In an earlier paper, however, Bodian, Carter, and Ward (1951) cited 3 cases (Perrot and Danon, 1935; Tiffin, Chandler, and Faber, 1940; Swenson, Rheinlander, and Diamond, 1949) in which there were normal ganglion cells both above and below the aganglionic colonic segment. $\mathrm{He}$ regarded these as rare variants of Hirschsprung's disease. Further sporadic reports of similar atypical Hirschsprung's disease with zonal aganglionosis have been reported by Keefer and Mokrohisky (1954), Hiatt (1951), and Pagès and Duhamel (1966).

In a review of Hirschsprung's disease treated surgically in the Radcliffe Infirmary between 1950 and 1970, we have encountered 4 similar cases. These are reported in this paper because our experience indicates that this atypical variant of Hirschsprung's disease is not as uncommon as has been suggested and may present problems in diagnosis and management.

\section{Methods}

All tissues were fixed in formol-saline and processed through paraffin. The biopsy specimens were embedded

Received 20 September 1971. so that sections were obtained at right angles to the mucosal surface, and step serial sections were stained with haematoxylin and eosin. Blocks of tissue which included the whole cross-section of the bowel were taken from the colectomy specimens at frequent intervals along their length. All tissues included both submucous and intermyenteric plexuses.

Case 1. A firstborn female, born 14 June 1970, whose father also had a history of Hirschsprung's disease. Feeds were taken early and vomited back and the first stool was not produced until 5 days. No further stool was passed and she was admitted to hospital with faeculent vomiting. The abdomen was grossly distended and a rectal examination revealed mucosal prolapse and an area of resistance at $3 \mathrm{~cm}$ from the anal margin.

A decompression colostomy was carried out with good result and colonic biopsies were taken at this time. At $4 \frac{1}{2}$ months she was readmitted and a Rehbein resection and 'pull-through' operation was performed.

The postoperative course was relatively satisfactory apart from persistent diarrhoea and rectal stricture which necessitated dilatation 2 months after operation. The patient still has bouts of diarrhoea but is gaining weight.

Histopathology. Initial full thickness biopsies from the rectosigmoid junction and colostomy site revealed aganglionosis in the former and ganglion cells in the latter.

The resected segment of sigmoid colon and rectum contained ganglion cells in apparently normal numbers in both intermyenteric and submucous plexuses in the distal segment, though their cytoplasm was sometimes 
vacuolated. An aganglionic segment was present in the sigmoid colon with abnormal nerve trunks in both plexuses. This segment extended proximally to the colostomy in the lower descending colon.

Case 2. A firstborn male, delivered on 11 July 1967, started to have abdominal distension with vomiting at 24 hours after birth. On the second day a meconium plug was passed per rectum followed by mucus and the abdominal distension decreased. Subsequent rectal examination revealed no further meconium and an abdominal $x$-ray confirmed persistent gaseous bowel distension. He was eventually discharged without treatment, but was readmitted one year later with a history of persistent constipation and one bowel action every 6 days.

He was given bowel washouts, and at laparotomy, 2 months later, when a defunctioning colostomy was performed, a coned sigmoid colon was noted and biopsies were taken from colostomy site and the sigmoid colon.

One year later a colonic resection and Rehbein 'pull through' operation were performed. Occasional episodes of diarrhoea occurred postoperatively and an anal stricture was twice dilated.

Histopathology. The initial full thickness biopsies revealed an aganglionic segment in the sigmoid colon with large abnormal nerve trunks. The colostomy site appeared normal.

$20 \mathrm{~cm}$ of resected colon revealed an absence of ganglion cells at the junction of sigmoid colon and

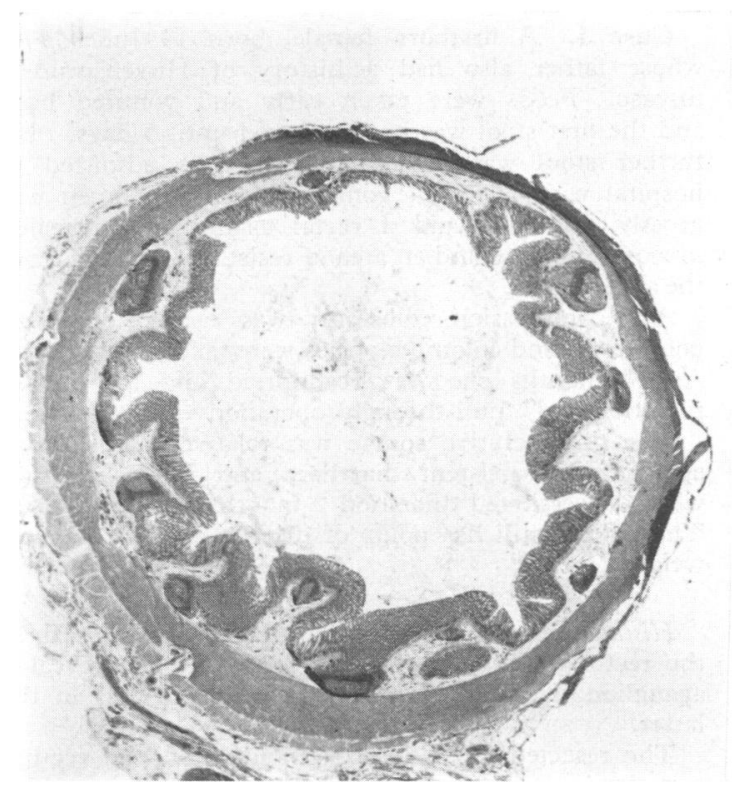

rectum with reappearance of these cells both proximally and distally. No apparent transition zone was present.

Case 3. A male, born 19 September 1952, at 4 days started to have bile-stained vomitus with abdominal distension. A right inguinal colostomy was performed.

Two years later a rectosigmoidectomy with closure of colostomy was carried out, but he had to have several subsequent admissions because of intestinal obstruction due to peritoneal adhesions.

In 1955 a further laparotomy revealed thickening of transverse and descending colon, and in February $\vec{\omega}$ 1956 a defunctioning transverse colostomy was performed. 3 months later he had a further resection of the terminal $25 \mathrm{~cm}$ of colon, and continuity was reestablished $8 \mathrm{~cm}$ from the anus-proximal to the previous anastomosis-with closure of his colostomy.

Further bouts of subacute intestinal obstruction ? necessitated several readmissions, these being thought $N$ to be due to peritoneal adhesions and a chronic volvulus. $\omega$

$\mathrm{He}$ was transferred to another hospital, and his subsequent progress is not available.

Histopathology (Fig. 1 to 3). Only sections from the rectosigmoidectomy specimen removed in 1954 were available; this was $20 \mathrm{~cm}$ long with a central colostomy.

An area of aganglionosis was present just distal to the colostomy with prominent abnormal nerve trunks in the intermyenteric and submucous plexuses (Fig. 3). Ganglion cells reappeared distally in normal numbers. A transition zone was found proximal to the colostomy.

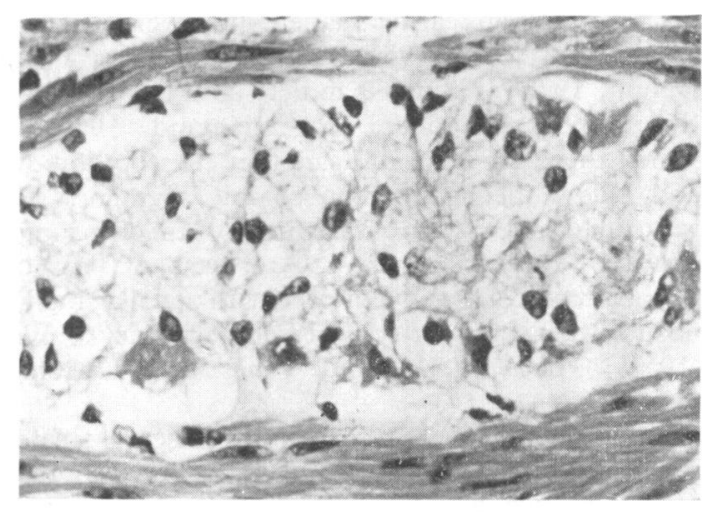

FIG. 1.-Case 3. Proximal segment showing (left) dilatation of colon $(H$. and $E$. $\times 4)$, and (right) ganglion cells in the intermyenteric plexus $(H$. and $E . \times 320)$. 

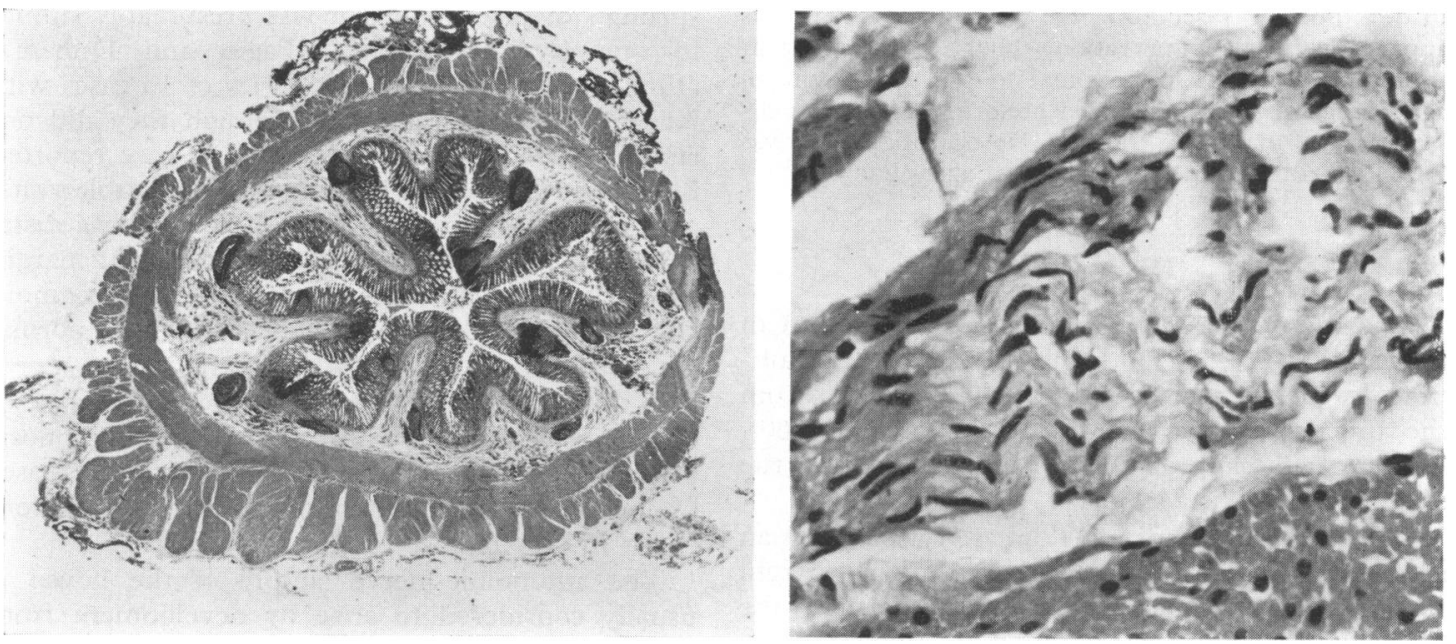

FIG. 2.-Case 3. Contracted segment of colon with absent ganglion cells and abnormal nerve trunks in the intermyenteric plexus.

Case 4. A male, born 20 September 1957, started to vomit at $\mathbf{5 6}$ hours when lower abdominal distension was noted. He had not had a bowel action at 4 days when a rectal washout produced flatus and faeces. After this his bowels opened normally for a few days, but then vomiting with constipation alternated with bowel actions. 2 months later a transverse colostomy was performed and the colon was noted to be thickened.

$\mathrm{He}$ was subsequently discharged and maintained normal progress with good weight gain despite further episodes of vomiting with constipation.

One year later he was readmitted with inversion of his colostomy, and biopsies of colostomy site and rectum were performed. The colostomy subsequently everted spontaneously but was not functioning satisfactorily, and 6 months later he was admitted for rectosigmoidectomy and reanastomosis. He progressed well though anorectal dilatation had to be performed on 2 subsequent occasions.

Histopathology. No ganglia were present in the initial full thickness biopsies of colostomy site and rectum.

$15 \mathrm{~cm}$ of excised sigmoid colon and rectum contained 2 areas of aganglionosis with prominent abnormal nerve trunks. The distal aganglionic segment extended
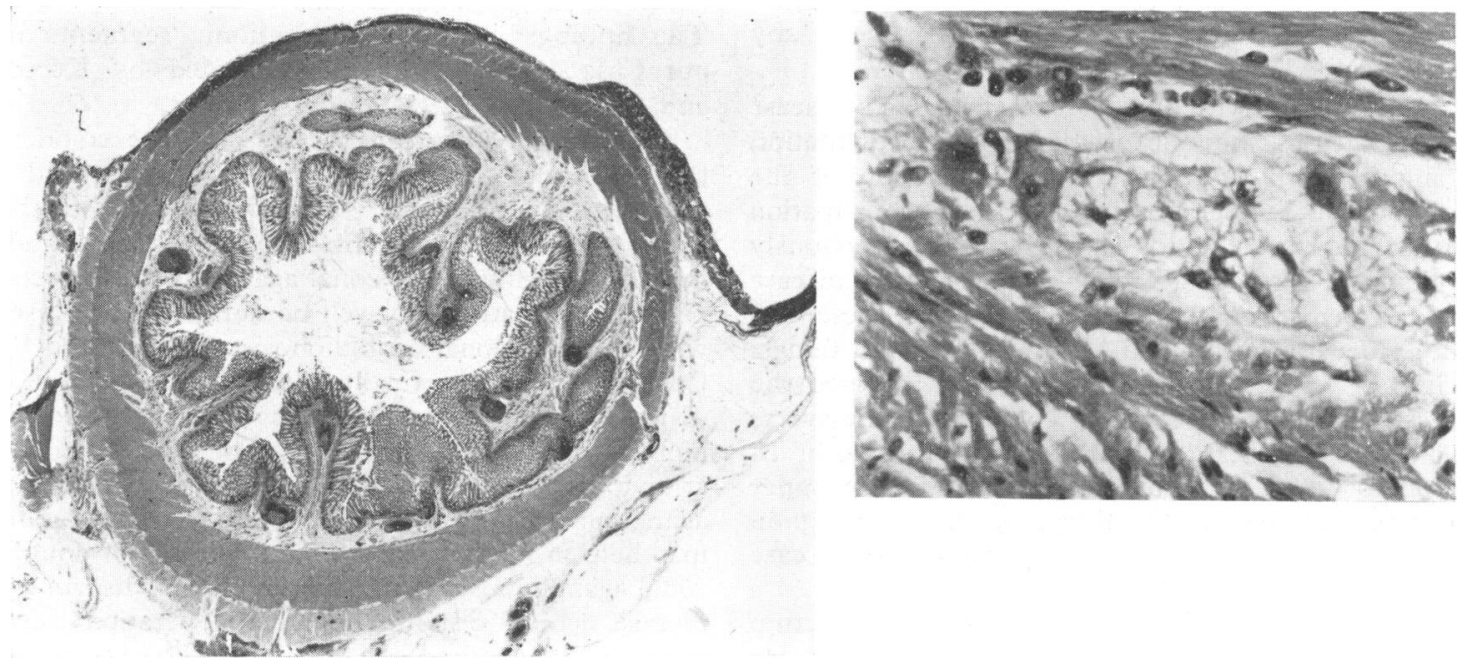

FIG. 3.-Case 3. Distal segment of colon with reappearance of ganglion cells in the intermyenteric plexus. 
to the line of resection. Between these there was apparently normal innervation, though a short transition zone was present proximal to the distal zone of aganglionosis, with vacuolated abnormal ganglion cells but no abnormal nerve trunks. No transition zone was found proximal to the upper zone of aganglionosis.

\section{Discussion}

The most distal ganglion cells can be found in the normal colon in both intermyenteric and submucous plexuses at a distance of 3-15 $\mathrm{mm}$ from the mucocutaneous anal margin (Bodian, 1960). They usually appear to be less frequent in the submucosal plexus.

In typical Hirschsprung's disease of the colon an area of aganglionosis extends from the anal margin for a variable distance proximally, though the majority involves the rectum and sigmoid colon only (Bodian, Stephens, and Ward, 1949). The aganglionic zone is characterized by the presence of nonmyelinated nerve trunks, abnormal both by their presence and size. A transition zone is found proximal to this which extends for a further variable distance until the colonic innervation becomes normal.

The transition zone contains a decreased number of ganglion cells and these are often smaller than normal with vacuolation of their cytoplasm. Abnormal nerve trunks are not present except at the junction with aganglionic areas.

In the first 3 cases reported above, the aganglionic segment did not extend to the anus, and in the fourth case there were 2 separate zones of aganglionosis - the distal one extending to the anal margin. In only 2 cases was there a transition zone of any appreciable length.

In common with typical Hirschsprung's disease these 4 cases show a varying clinical presentation ranging from acute intestinal obstruction of the newborn in 3 to persistent and recurrent constipation in the fourth. This is also true of the previously published cases of atypical Hirschsprung's disease with zonal aganglionosis. The first of these was described by Perrot and Danon (1935) in a female child of 15 days whose aganglionosis involved the terminal ileum, ascending colon, and transverse colon. Tiffin et al. (1940) reported a male of 18 months with a zone of aganglionosis in the upper sigmoid colon with a distal reappearance of ganglion cells. Swenson et al. (1949) referred to a third case of sigmoid aganglionosis. Hiatt (1951) reported a case with an aganglionic zone in the upper rectum with ganglion cells reappearing distally. $\mathrm{He}$ referred to this as the 'classical type' of Hirsch- sprung's disease, though it was presumably similar to our atypical variants. Pagès and Duhamel (1966) reported 2 cases in a series of 13 cases with failure of colonic propulsion though they did not state the site of aganglionosis. The case reported by Keefer and Mokrohisky (1954) of double zonal aganglionosis is similar to our Case 4 with a distal aganglionic segment extending to the anal margin and a proximal aganglionic segment in the sigmoid colon with an intervening zone containing normal ganglion cells.

It thus appears that most cases of this variant of Hirschsprung's disease have a zone of aganglionosis involving the sigmoid colon, and that the 2 cases of double zonal aganglionosis involved both sigmoid colon and rectum.

The autonomic nerve supply of the bowel is usually considered to arise by development from neuroblasts which have migrated from the neural crest, this development occurring in a craniocaudal direction. The submucosal plexus may develop later than the intermyenteric plexus (Spencer, 1966). The congenital absence of ganglion cells in Hirschsprung's disease may thus be due to an arrest of neuroblast migration or a failure of maturation with subsequent atrophy. The abnormal nerve trunks found in this disease are probably secondary to the aganglionosis. The presence of zonal aganglionosis with normal ganglion cells distally probably indicates that neuroblast migration or their subsequent development can proceed again following arrest. This seems a more likely explanation than the suggestion that these cases may be the result of a caudocranial development of ganglion cells (Bodian et al., 1951). The findings of double aganglionic segments in our Case 4 and in the case recorded by Keefer and Mokrohisky (1954) bears this out.

Atypical Hirschsprung's disease of the colon is thus an important variant from a diagnostic and a pathogenetic viewpoint. It may present in the varied manner of typical Hirschsprung's disease, and, in those showing single zonal aganglionosis, a rectal biopsy examination may well be normal. The cases with a double zonal aganglionosis as illustrated by Case 4 will present further difficulties. Even if a diagnosis of distal aganglionosis is made by rectal biopsy, the macroscopical appearances of the bowel at operation may not allow recognition of a second aganglionic zone. Multiple biopsies at operation may help in its location. The possibility of double zonal aganglionosis in those cases of Hirschsprung's disease which respond poorly to operation must therefore always be kept in mind. The incidence of 4 cases in a series of 15 cases at the Radcliffe 
Infirmary indicates that this variant is not as uncommon as has been previously suggested (Bodian et al., 1951).

\section{REFERENCES}

Bodian, M. (1960). Pathological aids in the diagnosis and management of Hirschsprung's disease. In Recent Advances in Clinical Pathology, Series III, p. 384. Ed. by S. C. Dyke. Churchill, London.

Bodian, M., Carter, C. O., and Ward, B. C. H. (1951). Hirschsprung's disease. Lancet, 1, 302.

Bodian, M., Stephens, F. D., and Ward, B. C. H. (1949). Hirschsprung's disease and idiopathic megacolon. Lancet, 1, 6.

Hiatt, R. B. (1951). The surgical treatment of congenital megacolon. Annals of Surgery, 133, 321.

Keefer, G. P., and Mokrohisky, J. F. (1954). Congenital megacolon (Hirschsprung's disease). Radiology, 63, 157
Pagès, R., and Duhamel, B. (1966). Intrinsic non-propulsive colon. Archives of Disease in Childhood, 41, 151.

Perrot, A., and Danon, L. (1935). Obstruction intestinale de cause rare, chez un nourrisson. Annales d'Anatomie Pathologique, 12, 157.

Spencer, B. (1966). Problems in rectal biopsy due to immaturity of ganglion cells. Archives of Disease in Childhood, 41, 149.

Swenson, O., Rheinlander, H. F., and Diamond, I. (1949). Hirschsprung's disease: a new concept of the etiology. New England Fournal of Medicine, 241, 551.

Tiffin, M. E., Chandler, L. R., and Faber, H. K. (1940). Localized absence of the ganglion cells of the myenteric plexus in congenital megacolon. American fournal of Diseases of Children, $\mathbf{5 9}, 1071$.

Correspondence to Dr. A. G. MacIver, Harkness Laboratory, Department of Pathology, Radcliffe Infirmary, Oxford. 